\title{
Collaborating and communicating Humanities scholars working and talking together
}

A

mong the academic truths that we generally hold to be self evident, are 1) the inherent value of collaboration and 2) humanists tend to be lone scholars, tucked away at their desks or in their carrels, surrounded by their books and papers, jealously guarding their intellectual expression until such a time as it can spring from their heads, fully formed, into the world. Like all truisms, these are open to dispute. Anyone who has tried managing projects undertaken by those with a diversity of personalities and perspectives, intellectual and otherwise, can quickly summon examples of the sometimes chaotic inefficiency of collaboration undermining the benefits afforded by that diversity. More positively, one can assert that those lone scholars in their studies are always working in collaboration, often across time and space, through the mediation of texts, rather than in team meetings and group conversations.

I advocate for and have my own experience of the value of scholarly collaboration, a value arising from the interplay of perspectives and expertise made possible by collaboration and from the efficiency engendered by a suite of complementary strengths. Collaboration is having a (extended) moment in the humanities; lone scholars are being lured or driven from their ivory towers, out onto the intellectual commons. Collaboration in the humanities is on the ascent, by necessity and design.
The necessity is often technology driven, as humanists become fully resident in the digital landscape and need to learn and deploy tools that ensure success there, an imperative that means engaging with others as mentors, tutors, and partners. Networked communication also makes possible the discovery of others who share interests and expertise and enables relationships with like-minded scholars who may live across the globe, state lines, or in a department across campus. As the institutional pressure to engage students at all levels in scholarship and research increases, a relatively new kind of student-faculty collaboration is also appearing in the humanities.

Humanities collaboration evidences itself most clearly in the work and inquiry known as the Digital Humanities. Digital Humanities tend to be self-consciously collaborative. Increasingly, this collaborative philosophy is also appearing elsewhere in the humanities community. Indicative of this shift is the Humanities Without Walls (HWW) initiative, funded by the Andrew W. Mellon Foundation. HWW, as it is familiarly known, exists to link 13 of the institutions, and their humanities centers, that belong

Maria Bonn is senior lecturer, School of Information Sciences at the University of Illinois at Urbana-Champaign, email: mbonn@illinois.edu

(C) 2017 Maria Bonn 
to the Big Ten Academic Alliance. HWW, by its own description, "aims to create new avenues for collaborative research, teaching, and the production of scholarship in the humanities, forging and sustaining areas of inquiry that cannot be created or maintained without cross-institutional cooperation." The initiative makes sub-awards to teams of humanities scholars that share a project but not an institutional location. The roughly 25 awards (as of publication) have required that the research teams include scholars from at least two of the consortial institutions.

I am both a beneficiary and student of the HWW effort. I am part of a research team from the University of Illinois UrbanaChampaign and Indiana University, led by librarians Harriett Green and Angela Courtney. Our group received an HWW award to study Humanities Collaboration and Research Practices with a particular focus on other HWW awardees. Our goal is to better understand the benefits and challenges of collaborative humanities scholarship.

Both individually and as a team, my group is interested in how those benefits and challenges are articulated and experienced in light of the work of scholarly communication. The project team conducted semi-structured interviews with 28 researchers who participated in projects funded by the first round of HWW awards. In each interview, we asked about intentions and ambitions for publication and sharing, and what barriers impeded the realization of those ambitions. Our work has culminated in a final white paper, forthcoming, from which I draw liberally here.

When respondents were asked about formats for expressing and sharing project work, they named many: performances, films, and websites, as well as traditional written texts and academic presentations. A number of respondents envisioned using hybrid formats to express their research. One respondent, for example, described an intention "to create some kind of interactive map [and] ideally a repository of sounds." Another discussed strategies for sharing interview data as a form of dissemination, noting that "we're still processing the data [and] deciding how to feature it."

The variety of publication formats used or envisioned by the interviewed researchers suggests that scholars increasingly may break away from traditional journal articles and monographs to explore many other modes by which their scholarship can be represented and communicated. Respondents saw avenues for making a broader impact via dissemination through a variety of platforms. As one respondent explained, "I think what we've contemplated is public dissemination of research using new platforms. I think we've contemplated scholarly output in the traditional platforms . . . journals, whether they're online or in print, but we have contemplated getting research into the hands of stakeholders who are not scholars."

Scholarly communication is certainly intertwined with establishing and maintaining a professional reputation and record of productivity, a fact of the scholarly existence that was evidenced as respondents discussed collaborative initiatives. Many were mindful of the importance of providing appropriate credit and recognition for project partners. One respondent noted, "For us, the notion of collaboration was built around the idea that both parties would be equally acknowledged." Negotiating appropriate credit, however, also revealed moments of tension within projects. Another respondent observed that "there was a little bit of misunderstanding, and some disagreements [. . .] had to do with who is being acknowledged for what."

Respondents differed on whether they planned for their collaboration to culminate in coauthored publications. One respondent noted, "I didn't expect a lot of coauthoring; more of a codesign of the platform." Another viewed co-authorship as an important "end product collaboration."

While discussion of evaluation for tenure and promotion were present within 
the interviews, they were not as prevalent as might be expected. One respondent did note that "Humanities have sort of a hard time understanding how to evaluate joint publications." In an anecdote about a colleague, another respondent described how coauthored publications in a tenure portfolio made the process of evaluation more difficult, but did not ultimately impede the scholar's promotion.

We also inquired about the intended venues for publication. Respondents saw their work as being published in multiple outlets, often in ways that evolved with the project itself. One respondent described having multiple presentation paths: "Initially it's a web presence, but I can see process papers coming out of this in more humanities journals." Other places where respondents published and disseminated their works included museum exhibitions, cinemas, YouTube, in the classroom, at conferences, and online.

For some, a digital platform also functioned as a publication venue: One respondent observed, "In my field at least, there are few kinds of places where scholarship, once published, then has a continuing life ... to me, one of the promises of Scalar ${ }^{3}$ existing online is that there is a potential for people to comment on and add to and use in different ways." This suggests that the ways in which audiences can interact with the scholarship and venues that facilitate interactivity may become increasingly important to scholars' publishing desires and needs.

Respondents drew upon both multimedia and analog forms to represent their data, findings, and ultimate project results. We found that the researchers frequently employed fluid and hybrid modes of representing their work, ranging from a combination of transitory performance, written choreography, and workshop to a blog that accompanied a larger project. One respondent described how new modes offered by digital tools and diverse media are critical to deepening humanities inquiry:
A lot of Humanities fields deal in some way in interpretation and trying to understand the different ways that we can think about all kinds of different texts and we've also been really limited in the Humanities in the peer review system to what we can tell about our projects. In most peer-reviewed journals, it's very hard to put pictures or video or, you know, further tell the story that we're trying to tell.

Respondents often considered whether and how their data and scholarship was reaching the intended audiences. One person observed, "This is the critical question when we look at new platforms and new forms of dissemination. Are they serving the technologies? Are they serving the institutions that get grants to build these digital archives and laboratories for this sort of thing or are they serving those that want to receive the materials themselves?"

There is was much more discussion throughout our interviews of scholarly communication writ large, ranging from methods for managing email to giving voice and credit to student collaborators in project publications. I would be remiss if, in reporting on our subjects' thoughts about innovation and change in scholarly communication, I lost sight of one other recurrent theme-the value of scholars meeting in person and communicating face to face.

Across the projects the amount of inperson communication varied from never having met prior to and never meeting during the work to spending a week together with shared dinners every night. We heard often that in-person meetings and occupying shared physical space strengthened and accelerated collaborative relationships and productivity.

Taken as whole, our interviews revealed a humanities culture very much aware that it is in a period of change in scholarly communication and a parallel change in 
the means and methods that best facilitate the scholarly conversation. This awareness very much came to the fore in the conversations created by collaboration.

One respondent reflected, "We're having a lot of discussion about print vs. digital in terms of the final platform for this thing. I think in some ways we sort of skip around to like the era of blogs and the era of listservs and these different kind of spaces that we think of through our scholarly careers that facilitated robust community interaction and exchange."

I began by asserting that academic culture tends to see the value of collaboration as self-evident. We found nothing in our investigation to dispute that belief. The collaborative experience was viewed positively and often enthusiastically by all of our respondents, an enthusiasm I have not shared as I use my space here to maintain a focus on the scholarly communication dimensions of humanities collaboration.

In our application for HWW funding, my team promised recommendations for supporting and sustaining humanities collaboration. In order to share the outcomes of such collaboration most effectively and create impact, we suggest that there are things that individual scholars and disciplinary and institutional cultures should do to ensure a more stable and supportive climate for humanities collaboration. These include experimenting with new forms, venues, and methods of dissemination that more accurately convey the full breadth of collaborative work and developing a culture that recognizes and rewards the value of that experimentation.

In addition, both individual scholars and the organizations of which they are a part should encourage a culture of sharing data and interim phase research within the humanities, opening the humanities process to others, and welcoming guidance from those others in conducting that process.

As my research team draws its activities to a close, my own sense of collaboration itself as an important form of scholarly communication is considerably strengthened-it is embedded in and extends the core scholarly activities of learning, teaching, and sharing.

In that light, it is imperative for me to conclude by saying that while all opinions expressed in this column are my own, their formation and expression are extensively shaped by the work and ideas of my collaborators: Harriett Green (PI), Megan Senseney, and Justin Williams from University of Illinois at Urbana-Champaign; and Angela Courtney (PI), Robert McDonald, Nicholae Cline, Leanne Nay, and Jaimie Murdock from Indiana University-Bloomington. I thank them for their contributions reflected here and for their good fellowship throughout the research and writing process.

\section{Notes}

1. See "About," Humanities without Walls, accessed January 26, 2017, www. humanitieswithoutwalls.illinois.edu/about /index.html.

2. Please note that all quotations are included with consent in accordance with IRB approval of our research protocols.

3. Scalar is a free, open source authoring and publishing platform designed to make it easy for authors to write long-form, borndigital scholarship online. See https://scalar. usc.edu/scalar/. n

\section{Scholarly communication column news}

The new and updated ACRL Scholarly Communication Toolkit is now available at http://acrl.libguides. com/scholcomm /toolkit/.

Could you be the author of the next "Scholarly Communication" column? Tell us your ideas! https://is.gd /crlnscholcomm. 